\title{
Role of Imaging in Spontaneous Coronary Artery Dissection - A Report and Review of Literature
}

Jagadeesh K Kalavakunta, ${ }^{1}$ Mohammad Hajjar, ${ }^{2}$ Rakshita Chandrashekar, ${ }^{3}$ Yashwant Agrawal, ${ }^{3}$ Nandu Gourineni ${ }^{4}$ and Tim A Fischell ${ }^{5}$

1. Interventional Cardiolgist; 5. Professor, Department of Cardiology, Michigan State University/Borgess Medical Center, East Lansing, Michigan, US; 2. Fellow; 4. Interventional Cardiologist, Department of Cardiology, Michigan State University/Sparrow Hospital, East Lansing, Michigan, US; 3. Resident Physician, Department of Internal Medicine/Pediatrics, Western Michigan University, Kalamazoo, Michigan, US

S pontaneous coronary artery dissection (SCAD) is a rare cause of acute ischaemic coronary events. It usually occurs during pregnancy or in the post-partum period in young women who have no traditional risk factors for coronary artery disease. We present two cases of SCAD in post-partum young women, who presented acutely with chest pain. Both underwent coronary angiography after worsening cardiac biomarkers. We utilised intravascular ultrasound (IVUS) in the first case, and optical coherence tomography (OCT) in the second case in order to confirm the diagnosis, define the anatomy and to measure the lesion length. This also serves to review the current literature and the available guidelines for treatment options for SCAD. We conclude that it is important to suspect SCAD as a cause of acute coronary syndrome in young female patients who present with chest pain especially in the post-partum period. These case studies also suggest a valuable role for IVUS and/or OCT in suspected cases of SCAD. This imaging is helpful in confirmation of the diagnosis, and allows optimal and precise treatment.

\section{Keywords}

Spontaneous coronary artery dissection, intravascular ultrasound (IVUS), optical coherence tomography (OCT)

Disclosure: Jagadeesh K Kalavakunta, Mohammad Hajjar, Rakshita Chandrashekar, Yashwant Agrawal, Nandu Gourineni and Tim A Fischell have no conflicts of interest to declare. No funding was received in the publication of this article.

Compliance with Ethical Guidelines: All procedures were followed in accordance with the responsible committee on human experimentation and with the Helsinki Declaration of 1975 and subsequent revisions, and informed consent was received from the patients involved in these case studies.

open Access: This article is published under the Creative Commons Attribution Noncommercial License which permits any non-commercial use, distribution, adaptation and reproduction provided the original author(s) and source are given appropriate credit.

Received: 7 May 2015

Accepted: 11 June 2015

Citation: European Journal of Arrhythmia \& Electrophysiology, 2016;2(2):62-5

Corresponding Author: Jagadeesh K Kalavakunta, Borgess Heart Institute, 1521 Gull Road, Kalamazoo, Michigan, 49048, US. E: jkalavakunta@gmail.com
Chest pain evaluation during pregnancy or the post-partum period poses diagnostic and therapeutic dilemma. The differential diagnosis would include acute myocardial infarction, coronary spasm, dissection, myocarditis and non-cardiac chest pain. Spontaneous coronary artery dissection (SCAD) is a rare cause of acute ischaemic coronary events. ${ }^{1,2}$ It usually occurs during pregnancy or post-partum period in young women who have no traditional risk factors for cardiac disease..$^{1,2}$ We present two interesting cases of post-partum acute myocardial infarction due to spontaneous dissection.

\section{Case 1}

A 36-year-old Caucasian woman presented to the emergency department (ED) with complaints of chest pressure. She is a mother of two children and gave birth to a stillborn, full-term baby 5 days prior to the presentation. Her chest pain was similar to her heartburn in the past, but lasted longer. Past medical history was significant for gestational diabetes and acid reflux disease. She never smoked and has no family history of premature coronary artery disease (CAD). Her vitals were noted as blood pressure of $136 / 88 \mathrm{mmHg}$, heart rate of 102 beats/minute, respiratory rate of 20/minute with 99\% oxygen saturation on room air. Cardiovascular examination revealed regular rate and rhythm without any significant murmurs, jugular venous distension or carotid bruit. Initial laboratory data revealed troponin of $2.32 \mathrm{ng} / \mathrm{ml}$ (normal range $<0.03$ ), creatine phosphokinase (CPK) 1,523 U/I (normal range 5-190), brain natriuretic peptide (BNP) $253 \mathrm{pg} / \mathrm{ml}$ (normal range 0-100), creatinine $0.5 \mathrm{mg} / \mathrm{dl}$, white blood cell count $12.2 \mathrm{~K} / \mathrm{ul}$ (normal range 4.5-11) and haemoglobin $12.8 \mathrm{gm} / \mathrm{dl}$. The chest X-ray was negative for any acute process. A 12 lead electrocardiogram (ECG) showed normal sinus rhythm with subtle ST segment elevation $(<0.5 \mathrm{~mm})$ and T wave inversions in V5 and V6 leads. She was treated as having an acute coronary syndrome (ACS). Since her symptoms improved upon arrival to the ED, she was not taken for an emergent cardiac catheterisation. However, the next day she had recurrence of chest pain along with elevation of troponin to $102 \mathrm{ng} / \mathrm{ml}$, CPK elevation to $3,452 \mathrm{U} / \mathrm{l}$ and her ECG demonstrated $1 \mathrm{~mm}$ of ST-elevation in the inferior and lateral leads suggestive of acute myocardial infarction (see Figure 1). Due to the symptoms, elevated cardiac biomarkers and abnormal ECG she was taken emergently to the cardiac catheterisation lab. Coronary angiogram showed angiographically normal coronary arteries except for a stenosed segment in the obtuse marginal (OM) branch of the left circumflex artery with varying severity and ulcerated appearance in the mid-portion of the stenotic segment. The most severe area of stenosis in the OM was visually estimated to be a 70-80\% diameter stenosis (see Figure 2). Distal to the stenosis, the OM branched with no narrowing in the branch vessels. Left ventricular angiography demonstrated severe hypokinesis of the lateral and infero-lateral segments, with an ejection fraction of $40-45 \%$. 

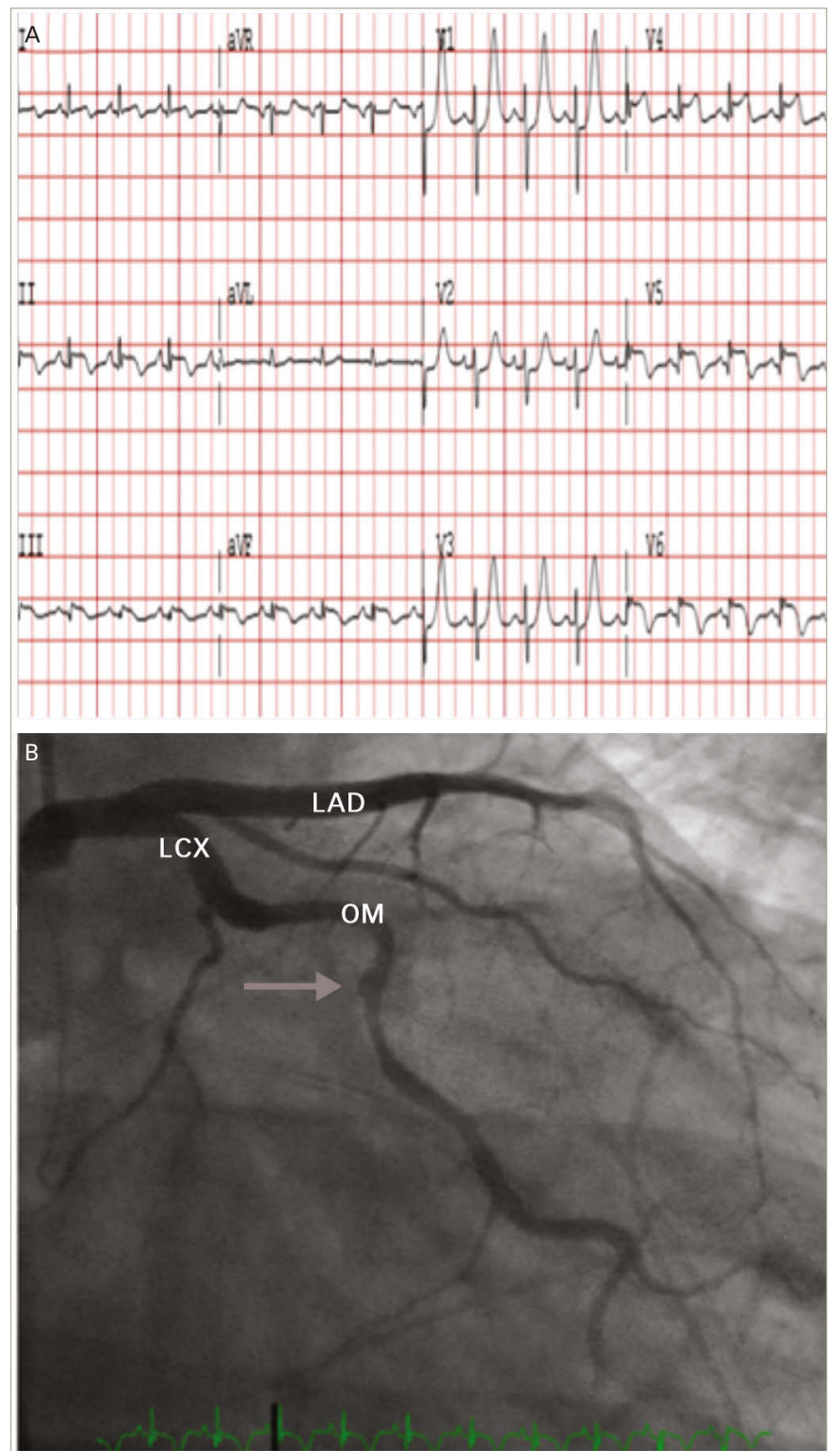

A: Electrocardiogram showing ST-elevation in the inferior and lateral leads (II, III, aVF, V4-V6); B: Left coronary angiogram in right anterior oblique and caudal view 300 showing the ulcerated lesion (arrow) in the obtuse marginal (OM) artery of left circumflex artery. $L C X=$ left circumflex artery; $L A D=$ Left anterior descending artery.

Since the patient did not have atherosclerotic plaques elsewhere, and the appearance was consistent with a possible spontaneous dissection, we elected to first study the OM with intravascular ultrasound (IVUS) to evaluate the underlying pathology. A 6F CLS 3.5 Runway guide catheter and an Asahi Prowater wire (Abbott Vascular, Santa Clara, California, US) was advanced smoothly across the stenotic lesion in the OM and advanced without difficulty to the distal segment. The IVUS probe was advanced distal to the lesion and an automatic pullback was performed per standard protocol. Images showed extensive dissection involving the OM branch and proximal circumflex artery with no appreciable atherosclerotic plaque. There was a tear noted in the mid-segment of the OM and extensive area of intramural haematoma throughout the entire segment of the stenosis (see Figure 3). The haematoma had the appearance of partially clotted blood in some regions. Revascularisation was then performed with direct stenting, utilising three XIENCE $\vee$ (everolimus eluting coronary stent, Abbott Vascular, Santa Clara, California , US) drug-eluting stents (DES). The final angiogram revealed a satisfactory result with good stent deployment and with thrombolysis in myocardial infarction (TIMI) grade 3 flow. After

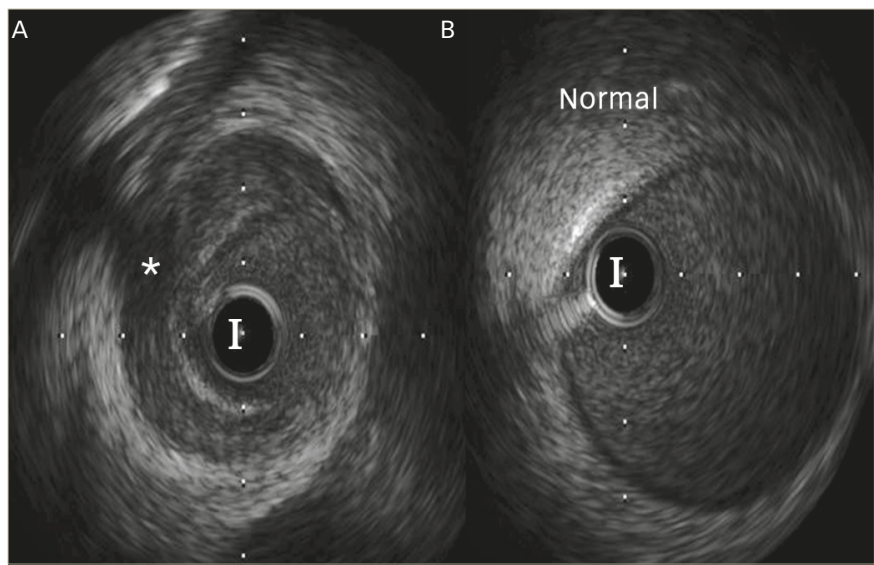

A: extensive dissection of the obtuse marginal artery with the intimal flap and intramural hematoma (*). B: Image of the normal artery for comparison. I = IVUS probe.

stenting, IVUS showed full apposition of the stents to the wall along the entire length of stenosis. Her post-cardiac catheterisation course was uncomplicated. She was started on aspirin, clopidogrel and beta-blockers. She continued to do well at follow up outpatient visits without any recurrence of symptoms.

\section{Case 2}

A 35-year-old female who was 7-day post-partum presented to the ED with 'ripping and tearing' retrosternal chest pain at rest that lasted for an hour. During her pregnancy she was diagnosed with pre-eclampsia and gestational diabetes. Other risk factors include family history of premature $C A D$ and 10-pack-years of smoking. On arrival to the $E D$ she was haemodynamically stable with heart rate of 85 beats/minute, blood pressure of $143 / 88 \mathrm{mmHg}$ and temperature of $98^{\circ} \mathrm{F}$. Her physical exam was unremarkable except for mild obesity. Initial ECG was within normal limits. Laboratory data were significant for troponin of $0.5 \mathrm{ng} /$ $\mathrm{ml}$ (normal range $<0.03$ ), and high sensitive C-reactive protein (hS-CRP) of $1.2 \mathrm{mg} / \mathrm{dl}$ (normal range $<3 \mathrm{mg} / \mathrm{dl}$ ). Her other laboratory results were unremarkable. She was admitted to the hospital and her chest pain was treated with analgesics. She did not have recurrence of chest pain after she arrived to the floor. Repeated cardiac enzymes showed a troponin level of $6.41 \mathrm{ng} / \mathrm{ml}$ (normal range $<0.03$ ). At approximately the time that the second troponin was completed, she became symptomatic with chest pain. At this point, treatment for acute non-ST elevation myocardial infarction (NSTEMI) was initiated with intravenous heparin, oral beta-blockers and a statin. She received aspirin in the ED, which was continued. She underwent urgent coronary arteriography, which showed a $75 \%$ diameter stenosis of the mid-portion of the left anterior descending (LAD) artery. The angiographic appearance was suggestive of a spontaneous dissection, with no apparent atherosclerotic lesions in other vessels. Left ventricular angiography showed minor anterior wall motion hypokinesis, but with a well-preserved overall ejection fraction of 55\%. A 6 French CLS 3.5 Runway guide catheter was placed in the left main coronary artery and a 0.014 Balance Middle Weight wire was advanced across the lesion and into the distal LAD. Optical coherence tomography (OCT) (St Jude Medical, Saint Paul, Minnesota, US) assessment of the mid-segment of the LAD using standard technique demonstrated an extensive dissection of the mid-LAD, associated with an intramural haematoma. The lesion was treated with direct stenting using a $3.5 \times 38$ mm Xience Xpedition (everolimus eluting coronary stent, Abbott Vascular, Abbott Park, Illinois, US) DES. Following placement of the first stent from in the mid-LAD, the proximal segment still appeared hazy. Therefore, OCT evaluation was repeated and showed that the lumen was 
Figure 3: A) Electrocardiogram; B) Right anterior oblique cranial view of the left anterior descending artery
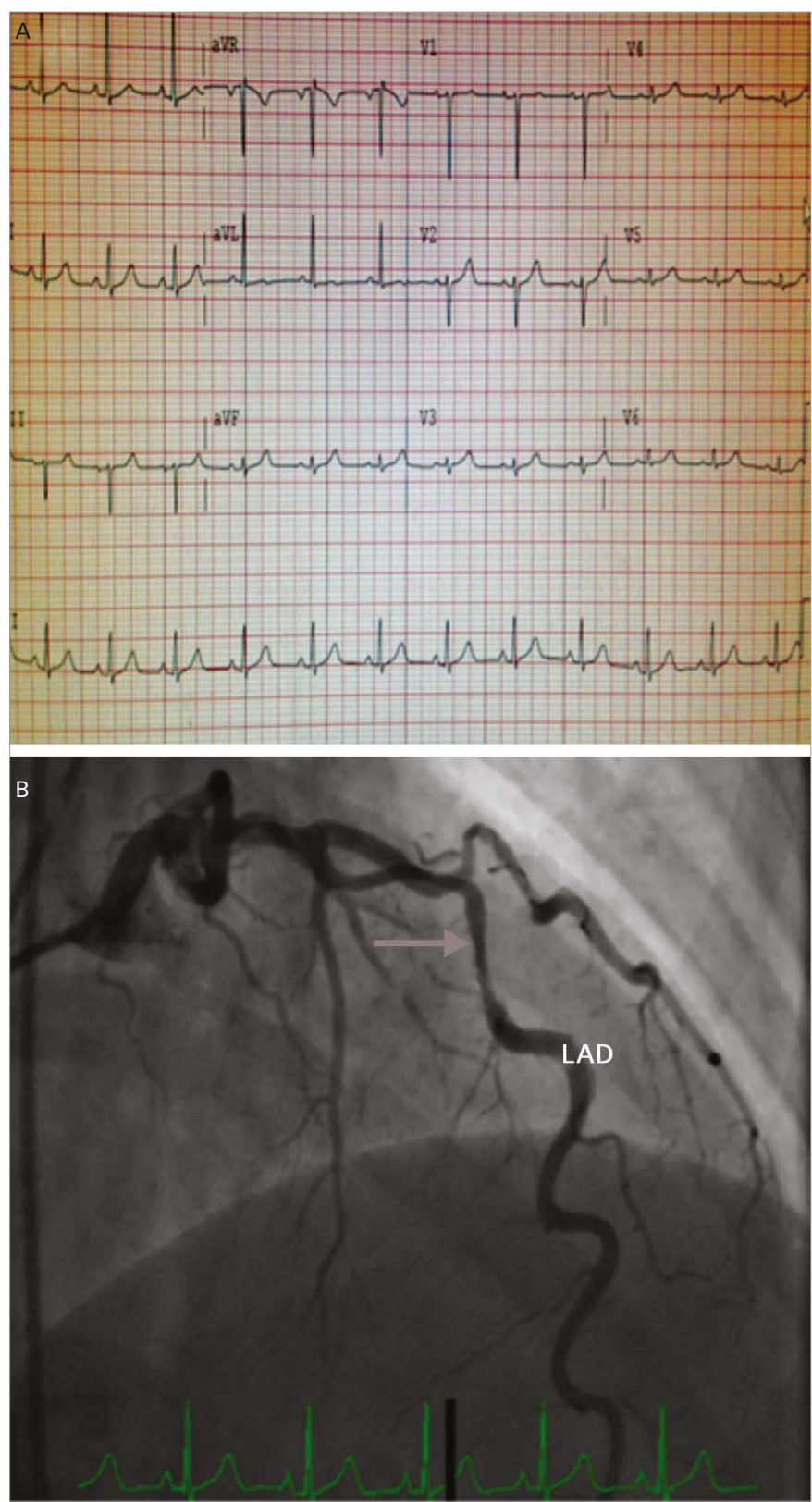

A: A 12 Lead electrocardiogram with normal sinus rhythm and without any acute ST-T changes suggestive of ischemia or infarction. B: Right anterior oblique (RAO) cranial view of the left anterior descending artery (LAD) showing smooth appearance of the stenosed segment (arrow) in the mid $L A D$.

clearly narrowed, despite some angiographic improvement with multiple doses of intra-coronary nitroglycerin and verapamil. It was suspected that a subintimal haematoma was propagating proximally and compromising the lumen, even though there was no clear dissection plane visualised. After the OCT evaluation, it was elected to place a second stent $(3.5 \times 23$ $\mathrm{mm}$ Xience Xpedition) from the proximal edge of the distal stent back to the ostial portion of the LAD. The final angiograms revealed TIMI grade 3 flow without any dissection, thrombus or significant luminal narrowing. Her post-procedure course was uneventful and she did very well at 1 month and 6 months follow up.

\section{Discussion}

SCAD is an extremely rare cause of ACS, with a reported angiographic incidence of $0.1-1.1 \% .^{1}$ It usually presents as angina pectoris or acute myocardial infarction, and is also associated with sudden cardiac death
Figure 4: Optical coherence tomography showing dissection

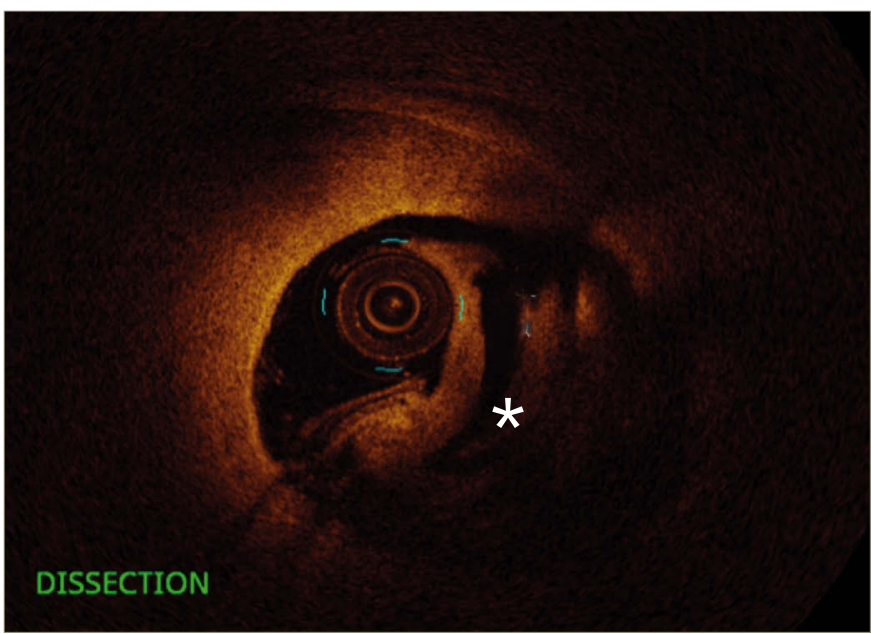

*of the left anterior descending artery with the intimal flap.

Coronary artery dissection can also occur as an extension of the proximal aortic dissection or iatrogenically due to cardiac catheterisation, coronary angioplasty, cardiac surgery and cardiopulmonary resuscitation.

SCAD has a significant female predominance, and is most commonly seen during pregnancy, and up to 12 weeks post-partum. ${ }^{1}$ It has also been described in association with trauma, exercise (more common in men), hypertension, cocaine abuse, fibromuscular dysplasia (FMD), cystic medial necrosis, contraceptive usage and in patients with various connective tissue and vasculitis disorders. Recently there was great interest in association between non-atherosclerotic SCAD and FMD. A study of 50 SCAD patients by Saw et al., found 43 (86\%) patients with $\geq 1$ non-coronary FMD suggestive of possible causative role in SCAD pathogenesis. ${ }^{2}$ The SCAD caused by atherosclerotic disease has to be distinguished from true SCAD. As described by Vrints, in most cases of atherosclerotic SCAD, the mild atherosclerotic plaque triggers rupture of the tunica intima, leading to tunica media dissection. ${ }^{3}$

Single coronary artery involvement is the most common presentation, but SCAD may also present with two or more vessels simultaneously. In a large Canadian series, they found $12 \%$ of patients had multivessel coronary dissection. ${ }^{2}$ Multi-vessel involvement confers poorer prognosis. SCAD most commonly involves the LAD artery (75\%) but has also been known to occur in other coronary arteries (right coronary artery $20 \%$, left circumflex $4 \%$ and left main $<1 \%) .{ }^{4}$ In our first case, $\mathrm{OM} /$ left circumflex artery was involved and, in our second case, the LAD was involved.

Although it has been more than 85 years since the first description of SCAD, the pathophysiology of this rare entity is not well understood. ${ }^{5}$ Several hypotheses have been proposed, such as coronary vasospasm, hypercoagulability, rupture of vasa vasorum, haemodynamic and hormonal changes during pregnancy, impaired collagen synthesis along with eosinophilic vasculitis and proteases secretion leading to degenerative changes in the vessel wall architecture. ${ }^{-9}$ SCAD can be unifocal or multifocal. In dissection of the coronary artery the tunica media is usually separated by blood with or without an intimal tear. The dissection flap or the enlarging haematoma can compromise the coronary blood flow leading to myocardial ischaemia or infarction. Depending on the coronary flow limitation, patients can be asymptomatic, or it can manifest as stable angina, unstable angina, myocardial infarction, cardiogenic shock and/or sudden cardiac death. In one study the short- 
term mortality with SCAD was $43 \% .^{10}$ Patients who survived the initial acute phase had better survival (82\% at 3 years of follow up). ${ }^{11}$

There is no general consensus regarding the first line of treatment for SCAD. The available literature is conflicting but, generally, management decisions are determined by the clinical picture and coronary angiographic evaluation. During the acute phase, dual antiplatelet agents and heparin are utilised along with beta-blockers/calcium channel blockers. The usage of glycoprotein IIb/Illa antagonists is controversial and usually avoided. ${ }^{12}$ In patients who have survived the acute phase, medical management alone may be sufficient. In one study of 45 consecutive SCAD patients, conservative management yielded good long-term prognosis with $92 \%$ event-free survival at 3 years. ${ }^{13} \mathrm{~A}$ recent observational study of 134 patients revealed a conservative approach had better in-hospital outcomes, but long-term outcomes were not different compared with revascularisation. ${ }^{14}$ In the same study, angiographic findings of distal location compared with proximal/mid and TIMI flow of 2/3 compared with 0/1 favoured conservative management with odds ratio of 9.27 and 0.20 , respectively. ${ }^{14}$

In patients with ongoing symptoms of ischaemia and single vessel coronary artery involvement, primary angioplasty with stenting has been shown to be effective, as was observed in our cases. A metaanalysis of 440 cases showed early intervention of SCAD led to better outcomes and fewer reinterventions..$^{15}$ Conversely, a Mayo clinic study of 87 patients showed percutaneous coronary intervention (PCI) had higher rates of technical failures (35\%) and did not protect against target vessel revascularisation or recurrence of SCAD. ${ }^{16}$ There is a limited amount of data available regarding the use of DES versus bare metal stents or bioabsorbable scaffolds in SCAD. Post-partum safety and contraindications of anticoagulation/antiplatelet agents need to be considered. DES may have superiority by reducing the neointimal growth and restenosis. Theoretically, $\mathrm{PCl}$ with stent placement provides benefit with immediate results by providing mechanical scaffolding and resolution of dissection. However, complications of $\mathrm{PCl}$ such as stent implantation in the false lumen, coronary perforation or worsening of the dissection need consideration in the clinical decision-making process. Current American College of Cardiology/ American Heart Association (ACC/AHA) guidelines recommend aggressive therapy with a caution regarding potential complications. ${ }^{17}$ Balloon angioplasty with cutting balloons, ${ }^{18}$ thrombolytic agents, immunosuppressive agents, ${ }^{19}$ bypass surgery, left ventricular assist device implantation and even heart transplantation have been utilised to treat SCAD successfully. ${ }^{20}$ The role of fibrinolytic therapy is controversial and it may be detrimental by worsening the intramural haematoma. ${ }^{21}$ Coronary artery bypass surgery should be considered in left main or/multi-vessel involvement, and possibly in cardiogenic shock patients. ${ }^{22,23}$

Utilisation of IVUS and OCT has made the diagnosis of SCAD and $\mathrm{PCl}$ with stenting more accurate. Multi-slice computed tomography may also be utilised in the diagnosis of SCAD in non-acute clinical scenarios. During angiography, the dissection appears as a radiolucent intimal flap or slow clearance of contrast from the false lumen. When there is no intimal tear, the haematoma appears angiographically as a stenosis, which may underestimate the dissection occurrence. IVUS and OCT help to differentiate dissections from atherosclerotic plaque, and delineates the exact lesion pathology in such cases. ${ }^{24}$ In one small study of eight SCAD patients, the combined use of IVUS and OCT was complementary. ${ }^{25}$ In our clinical practice, we noted similar findings as Paulo et al. noted in their study with OCT and IVUS in SCAD. Both imaging modalities have their own merits and limitations. OCT has approximately 10 times greater resolution compared with IVUS, which allows improved tissue characterisation and luminal-intimal interface delineation. ${ }^{26}$ IVUS provides complete vessel visualisation due to better penetration. Interventionalists should opt for either modality depending on their availability and comfort level in the utilisation. Post-procedural IVUS or OCT can confirm the appropriate sizing, positioning, apposition of the stent and also the successful sealing of the dissection.

In both of our cases, carrying out additional imaging not only helped to confirm the diagnosis of SCAD, but also helped to treat the entire lesion accurately, ultimately requiring more stents than what we might use based upon angiography alone. Our cases suggest that in clinical scenarios with suspected SCAD, it may be appropriate to have a lower threshold for cardiac catheterisation. In addition, early evaluation using IVUS and/or OCT should be considered, to not only help in the accurate diagnosis but also to guide therapeutic intervention.

\section{Conclusion}

SCAD is rare phenomenon. It should always be suspected as a cause of ACS in young female patients, especially during pregnancy or post-partum. Utilisation of IVUS and OCT in these scenarios can be very helpful to confirm the diagnosis, exact pathology and treatment effectiveness of $\mathrm{PCl}$. $\square$
1. Kamineni R, Sadhu A, Alpert IS, Spontaneous coronary artery dissection: report of two cases and a 50-year review of the literature, Cardiol Rev, 2002;10:279-84

2. Saw J, Ricci D, Starovoytov A, et al., Spontaneous coronar artery dissection: prevalence of predisposing conditions including fibromuscular dysplasia in a tertiary center cohort, JACC Cardiovasc Interv, 2013;6:44-52.

3. Vrints $\mathrm{CJ}$, Spontaneous coronary artery dissection, Heart, 2010;96:801-8.

4. Jorgensen MB, Aharonian V, Mansukhani P, Mahrer PR, Spontaneous coronary dissection: a cluster of cases with this rare finding, Am Heart J, 1994:127:1382-7.

5. Pretty $\mathrm{HC}$, Dissecting aneurysm of coronary artery in a woman aged 42: Rupture, BMJ, 1931;1:667.

6. Bonnet J, Aumailley M, Thomas D, et al., Spontaneous coronary artery dissection: case report and evidence for a defect in collagen metabolism, Eur Heart J, 1986;7:904-9.

7. Jessurun GA, Tio RA, Ribbert LS, et al., Unusual cause of sudden cardiac death: basophilic degeneration of coronary arteries, Cathet Cardiovasc Diagn, 1996;39:172-6.

8. Roth $A$, Elkayam U, Acute myocardial infarction associated with pregnancy, Ann Intern Med, 1996:125:751-62.

9. Verma PK, Sandhu MS, Mittal BR, et al., Large spontaneous coronary artery dissections-a study of three cases, literature review, and possible therapeutic strategies, Angiology, 2004;55:309-18.

10. Thompson EA, Ferraris S, Gress T, Ferraris V, Gender differences and predictors of mortality in spontaneous coronary artery dissection: a review of reported cases, I Invasive Cardiol,
2005:17:59-61.

11. Koul AK, Hollander G, Moskovits N, et al., Coronary artery dissection during pregnancy and the postpartum period: two case reports and review of literature, Catheter Cardiovasc interv, 2001;52:88-94.

12. Alfonso F, Bastante T, Cuesta J, et al., Spontaneous coronary artery dissection: novel insights on diagnosis and management, Cardiovasc Diagn Ther, 2015;5:133-40.

13. Alfonso F, Paulo M, Lennie $V$, et al., Spontaneous coronary artery dissection: long-term follow-up of large series of artery dissection: Iong-term follow-up of large series of therapeutic strategy, JACC Cardiovasc Interv, 2012:5:1062-70.

14. Lettieri C, Zavalloni D, Rossini R, et al., Management and long term prognosis of spontaneous coronary artery dissection, $A m$ J Cardiol, 2015;116:66-73

15. Shamloo BK, Chintala RS, Nasur A, et al., Spontaneous coronary artery dissection: aggressive vs. conservative therapy, I Invasive Cardiol, 2010;22:222-8.

16. Tweet MS, Hayes SN, Pitta SR, et al., Clinical features, management, and prognosis of spontaneous coronary artery dissection, circulation, 2012:126:579-88.

17. Amsterdam EA, Wenger NK, Brindis RG, et al., 2014 AHA ACC guideline for the management of patients with non-STelevation acute coronary syndromes: A report of the American College of Cardiology/American Heart Association Task Force on Practice Guidelines, J Am Coll Cardiol, 2014;64:e139-22.

18. Koller PT, Cliffe CM, Ridley DJ, Immunosuppressive therapy for peripartum-type spontaneous coronary artery dissection: case report and review, Clin Cardiol, 1998:21:40-6.
19. Yumoto K, Sasaki H, Aoki H, Kato K, Successful treatment of spontaneous coronary artery dissection with cutting balloon angioplasty as evaluated with optical coherence tomography, JACC Cardiovasc Interv, 2014;7:817-9.

20. Ferrari E, Tozzi P, von Segesser LK. Spontaneous coronary artery dissection in a young woman: from emergency coronary artery bypass grafting to heart transplantation, Eur J Cardiothorac Surg, 2005;28:349-35

21. Zupan I, Noc M, Trinkaus D, et al., Double vessel extension of spontaneous left main coronary artery dissection in young women treated with thrombolytics, Catheter Cardiovasc Interv, 2001:52:226-30.

22. Ooi A, Lavrsen M, Monro J, Langley SM, Successful emergency surgery on triple-vessel spontaneous coronary arter dissection, Eur J Cardiothorac Surg, 2004;26:447-9.

23. Samuels LE, Kaufman MS, Morris RJ, et al., Postpartum coronary artery dissection: emergency coronary artery bypass with ventricular assist device support, Coron Artery Dis, 1998:9:457-60.

24. Arnold JR, West NE, van Gaal WJ, et al., The role of intravascular ultrasound in the management of spontaneous coronary artery dissection, Cardiovasc Ultrasound, 2008:6:24.

25. Paulo M, Sandoval J, Lennie V, et al., Combined use of OCT and IVUS in spontaneous coronary artery dissection, JACC Cardiovasc Imaging, 2013;6:830-2.

26. Kubo T, Imanishi T, Takarada S, et al., Assessment of culprit lesion morphology in acute myocardial infarction: ability of optical coherence tomography compared with intravascular ultrasound and coronary angioscopy, I Am Coll Cardiol, 2007;50:933-9. 